\title{
Behavioral Psychology's Matching Law Describes the Allocation of Covert Attention: A Choice Rule for the Mind
}

\author{
Gene M. Heyman and Sebastian Moncaleano \\ Boston College
}

\begin{abstract}
The matching law describes the allocation of behavior over a wide range of settings, including laboratory experimental chambers, forest foraging patches, sports arenas, and board games. Interestingly, matching persists in settings in which economic analyses predict quite different distributions of behavior, and it also differs systematically from probability matching. We tested whether the matching law also describes the allocation of covert cognitive processes. Sixty-four participants viewed 2, small, vertically arranged adjacent stimuli that projected an image that fit within the fovea. A trial version of the reward contingencies used in matching law experiments determined which stimulus was the target. For example, in 1 condition, the top stimulus was the target 3 times more frequently than the bottom stimulus. However, the amount of time the stimuli were available was tailored to each participant so that they were not able to make use of the information in both stimuli even though an eye-tracking experiment confirmed that they saw both. The implication of this restriction is that participants had to decide which stimulus to attend to prior to each trial. The only available objective basis for this decision was the relative frequencies that a stimulus was the target. The matching law predicted the correlation between the relative frequency that a stimulus was the target and the proportion of trials that it was attended to. The results support the claim that the matching law is a general choice principle-one that describes the allocation of covert mental processes as well as overt behavioral responses.
\end{abstract}

Keywords: matching law, choice, attention allocation, covert attention, visual attention

Everyday language distinguishes between thought and behavior. Psychologists do the same. Their job titles, textbooks, and journal articles reflect the split between cognitive psychology and behavioral psychology. Nevertheless, thought and behavior share a fundamental property. Both are limited capacities. At any one moment there is more to consider than can be considered, and there is more to do than can be done. By definition, attention and choice are selective, and in both domains, reward plays a key role in what is selected (e.g., Chelazzi, Perlato, Santandrea, \& Della Libera, 2013). Our experiment follows from these observations. It tests whether a quantitative principle that guides choice under an extremely wide range of conditions also guides the allocation of cognition The choice principle has come to be known as "the matching law" (Herrnstein, 1970; Herrnstein, Rachlin, \& Laibson, 1997), and the experimental task that we use to test matching involves covert shifts in attention between two small, adjacent visual stimuli. There were no explicit rewards in the experiment, rather there was an objective relative rate of correct targets and, of course, the participant's judgment as to whether he or she had

AQ: 8 (D) Gene M. Heyman, Department of Psychology and Neuroscience, Boston College; Sebastian Moncaleano, Department of Measurement, Evaluation, Statistics and Assessment, Boston College.

Correspondence concerning this article should be addressed to Gene M. Heyman, Department of Psychology and Neuroscience, Boston College, 300

AQ: 9 McGuinn Hall, 140 Commonwealth Avenue, Chestnut Hill, MA 02467. Email: heymang@bc.edu made a correct response. Our analyses relied largely on the former measure. Thus, our experiment tests whether the matching law provides a precise quantitative description of the relationship between covert shifts in attention and the arranged frequencies of correct answers for such shifts. At a more general level, the experiment tests whether the matching law applies to the allocation of limited mental processes as well as it applies to the allocation of limited behavioral processes. To our knowledge, this is the first experiment that evaluates this question.

\section{The Matching Law}

According to the matching law, the overall distribution of choices approximates the overall distribution of rewards. For instance, if there are just two options, as is the case in many experimental studies, then

$$
B_{1} /\left(B 1+B_{2}\right)=R_{1} /\left(R_{1}+R_{2}\right),
$$

where $B_{i}$ is the frequency of choices 1 and 2 , and $R_{i}$ is the frequency of the rewards associated with choices 1 and 2 . Its simplicity notwithstanding, Equation 1 applies widely. Pigeons served as subjects in the initial experiment (Herrnstein, 1970). In subsequent experiments, matching was found in rats, monkeys, cows, humans, and even insects (e.g., Davison \& McCarthy, 1988; De Carlo \& Abramson, 2012; Grace \& Hucks, 2013). The settings have included but are not restricted to lever pressing in experimental chambers, signal detection tasks, foraging in natural settings, the opening moves in chess games, two- versus three-point shots in college basketball, and point-after-touchdown plays in the 
HEYMAN AND MONCALEANO

NFL (e.g., Cero \& Falligant, 2020; Falligant, Boomhower, \& Pence, 2016; Houston, 1986; Vollmer \& Bourret, 2000). The rewards have included but are not restricted to food, money, brain stimulation, drugs, alcohol, and social approval (e.g., Conger \& Killeen, 1974). This generality has attracted the interest of social psychologists (Gray, Stafford, \& Tallman, 1991), clinicians (Borrero \& Vollmer, 2002), criminologists (e.g., Rees \& Winfree, 2017), behavioral economists (Foxall, James, Oliveira-Castro, \& Ribier, 2010) and neuroscientists (Arvanitogiannis \& Shizgal, 2008; Soltani \& Wang, 2006). There are studies on the neural bases of matching (Loewenstein, Seung, \& Newsome, 2006) and an extensive literature on the relations between the matching law and maximizing as understood in biology and economics (e.g., Heyman \& Luce, 1979; Houston, McNamara, \& Steer, 2012; Kubanek, 2017). This high degree of generality helps explain why Equation 1 is often referred to as a "law." But in the hundreds of matching law studies the dependent variable was invariably a manifest activity, an action that could be videotaped.

\section{A Procedure for Quantifying the Allocation of Covert Attention}

The current experiment builds on two earlier studies from our laboratory (Heyman, Grisanzio, \& Liang, 2016; Heyman, Montemayor, \& Grisanzio, 2017). In these studies, a computer monitor displayed two, small, adjacent stimuli. A fixed probability determined which one was the target (as in probability matching studies), and, accordingly, the participant's task was to report on the contents of this stimulus. Although the two stimuli projected an image that did not exceed the dimensions of the fovea, (e.g., Wandell, 1995), which we confirmed in an eye-tracking study, (Heyman et al., 2017), the stimulus duration times were tailored to each participant so that he or she could report correctly on one stimulus, but not both. That is, the participants "saw" both stimuli but were able to identify the contents of just one. We developed a mathematical model of the procedure that allowed us to determine which stimulus they had actually attended. The equation included a term for correct guessing, and it was arranged so that the solution to the equation was the proportion of trials the participant had attended the target stimulus. That is, we calculated the allocation of covert attention.

A key feature of the procedure (and its mathematical model) was that the probabilities that the two stimuli provided the information necessary for a correct response summed to 1.0 in each trial. However, in most matching law experiments, two or more independent timers, each linked to one of the options, determines when a choice produces a reward. At any instant, one, both or neither option provides access to the rewards, and these probabilities necessarily increase as a function of time since the last obtained reward (in effect, they replenish). We modified the probability approach of the previous studies so that it mimicked the temporal, rate-based reinforcement contingences in matching law studies. As in matching law studies, on any given trial, one stimulus, both stimuli, or neither stimulus were the targets, and although a trial procedure, a clock-like probability device determined whether a stimulus was a target. For instance, as in matching law studies, the likelihood that a stimulus was the target increased as a function of the number of trials since the last correct choice; see the Method section for details (notice that this proce- dure differs from procedures in which the probabilitie: Th: $t$ the stimulus is correct remains fixed across trials; e.g., Shanks, Tunney, \& McCarthy, 2002; Shaw \& Shaw, 1977).

\section{Reward and Attention}

Over the last 20 years or so there has been growing interest in the degree and manner in which the stimulus's reward value influences attention (e.g., Anderson \& Yantis, 2013; Chelazzi et al., 2013; Lee \& Shomstein, 2014; Shahan \& Podlesnik, 2006; Small et al., 2005). In a particularly striking example, reward value undermined the much studied "attentional blink phenomenon" (Raymond \& O'Brien, 2009), and several studies show that stimuli that predict rewards can come to control attention in much the same manner as do highly salient physical features, such as abrupt onset times or striking color contrasts (e.g., Le Pelley, Vadillo, \& Luque, 2013). In a review of this literature, Chelazzi and his colleagues (2013) concluded with the sentence, "it may seem paradoxical that learning principles developed to explain overt behavior, within a theoretical framework that was skeptical about the hidden and impalpable intricacies of cognition ... appear... perfectly suited to account for reward-based changes in attentional priority." (p. 70). However, it is also the case that studies of how reward influences attention differ in a number of interesting ways from traditional behavioral studies. These differences include a strong interest in the neural correlates of attention and an interest in how different sources of stimulus salience combine to guide attention, whereby different sources, the researchers cite statistical learning, semantic relations, and reward (see, e.g., Chelazzi, Bisley, \& Bartolomeo, 2018; Jiang, 2018; Todd \& Manaligod, 2018). In the Discussion section, we point out that this theoretical orientation sheds light on an interesting feature of our results.

\section{Predictions}

We tested three quantitative hypotheses. First, that the matching law will predict the allocation of cognition about as well as it predicts the distribution of choices in behavioral studies. Second, we predict that the trial-by-trial shifts in attention will vary systematically as a function of the overall allocation of attention in a manner analogous to the relationship between moment-to-moment changes in preference and overall (e.g., session-wide) preference in behavioral experiments. Third, on the basis of our previous studies, we predicted that in trials in which the participants attended to the stimulus that provided the information necessary for a correct response, they will select the correct answer at least $90 \%$ of the time, whereas in trials in which the participants attended to the stimulus that did not provide the information necessary for a correct answer, they will perform at chance levels-which in this experiment was $14.3 \%$.

A caveat is in order. Our procedure and measures allow for more than one cognitive bottleneck. One stimulus may have left a weaker "initial trace," engendered weaker encoding, or, during the processing of the information in the attended to stimulus, the encoded initial trace of the other stimulus may have faded from memory. These are not mutually exclusive nor exhaustive possibilities. We say more about the constraints on cognition in the Discussion section of this paper. Put another way, when we use the word attention we are referring to the selective allocation of 
cognitive resources, which may or may not include the perceptualcognitive processes engendered by the experimental task.

\section{Method}

\section{Participants}

We tested 64 participants. Each completed two experimental sessions. The average age was 18.58 years $(S D=1.54) ; 45$ were women. Prior to the start of the experiment, participants signed a consent form according to the protocols established by the Boston College Institutional Review Board (IRB). The experimenter then read aloud a brief set of instructions (which were presented again on the monitor at the start of the study). The participants earned course credit for participating in the study. The procedure was approved by the Boston College IRB.

The number of participants was determined by our previous research with a similar version of the procedure used in the present study (Heyman et al., 2016). That experiment produced orderly and statistically significant results with 41 participants. We increased the number of participants in the present study in case the procedural changes led to more variable results.

\section{Equipment}

The experiment was conducted on a laptop computer. The screen measured $31.1 \mathrm{~cm} \times 17.5 \mathrm{~cm}$ and was set at a resolution of 1,366 pixels $\times 768$ pixels. The participants sat at their preferred distance from the computer, which varied from approximately 43 $\mathrm{cm}$ to $65 \mathrm{~cm}$.

\section{The Parameterized Matching Law}

On the basis of the first generation of matching law experiments, Baum (1974) introduced a more general, ratio version of Herrnstein's initial formulation (Equation 1). It included two free (fitted) parameters that captured systematic regularities in the data. We used this version to describe how the participants divided their attention between the two stimuli. In logarithmic coordinates, the reformulation of Equation 1 is a linear equation, in which the fitted, free parameters are the intercept and slope:

$$
\log \left(B_{1} / B_{2}\right)=\log a+b \log \left(R_{1} / R_{2}\right)
$$

According to much research, the intercept, $\log a$, measures asymmetries in the values of the alternatives, say a participant's side bias or systematic differences in the rewards (say, dollars vs. quarters), and the slope, $b$, reflects the degree to which participants accurately discriminate the rates of reward. (Baum, 1974, 1979). In most experiments, the bias parameter differs from zero and participants do not perfectly discriminate between the competing rates of reward.

\section{Key Features of the Procedure}

Figure 1 outlines the procedure. It shows the different displays that made up a trial, their order, and duration. The participant's task was to find the number in the response screen that was equal to the sum of the three digits in the top or bottom row of the stimulus screen. For example, in Figure 1, the three digits of the bottom row of the stimulus display add up to nine, and 9 is the fifth sum in the response display. Thus, the bottom row was the target stimulus, and the keyboard button labeled 5 was the correct response. What made this task challenging was that the duration of the stimulus screen had been tailored to each participant so that he or she had just enough time to reliably find the sum of one row of the stimulus display, but not enough time to find the sums of both rows (see the Calibration Procedure section). This means that the participants had to decide which stimulus to attend to prior to the start of a trial.

Whether the top or bottom stimulus was the target varied probabilistically. The nominal values expressed as overall ratios were $1: 9,1: 3,1: 1,3: 1$, and 9:1. For instance, in the $1: 9$ condition, the bottom stimulus was the target about nine times more frequently and conversely so in the 9:1 condition. Each participant experienced just one ratio and completed two sessions at this ratio. The participants learned the task readily but differed in terms of how much time the stimulus display needed to remain visible to correctly add the three digits of one row.

Calibrating the stimulus screen duration. At the start of each session, the participant completed a series of calibration trials. Our goal was to find the shortest stimulus display duration that supported correct responses. The calibration trials were organized into blocks of four, eight, and 16 trials. In three-quarters of the trials, a cue informed the participant whether the top or bottom stimulus was correct, with a $50 \%$ chance for one or the other. All participants were tested at exposure durations of $600 \mathrm{~ms}, 400 \mathrm{~ms}$, $300 \mathrm{~ms}, 200 \mathrm{~ms}$, and $150 \mathrm{~ms}$ in descending order in four-trial blocks. Next, we adjusted the exposure durations in smaller steps and increased the number of trials per block to eight or 16 . The adjustments continued until the participant correctly identified the number in the response display that corresponded to the sum of the three digits of the target stimulus at least $87.5 \%$ of the time in the cued trials, while performing at approximately chance levels in uncued trials.

Trial structure. Following the calibration procedure, the experimental session began. Each trial was initiated by a preparatory, countdown period, marked by asterisks that disappeared at a rate of $1 / \mathrm{s}$, with a $100-\mathrm{ms}$ interscreen interval (see Figure 1). Following the last asterisk, there was a 1-s screen that displayed the words Top or Bottom when it was a cued trial, or No Cue when it was a noncued trial. Next was the stimulus display; it remained on for the period determined by the calibration procedure-typically about $130 \mathrm{~ms}$ to $140 \mathrm{~ms}$.

The digits of the stimulus display were drawn from a list composed of the numbers 1 to 7 and were selected according to the following constraints: a row could not contain three instances of the same digit, the digits had to sum to a number on the interval 7 to 17 , each of the 11 possible sums had to appear equally often, and the sums associated with the top and bottom stimuli had to differ. Next the probe display asked the participant whether he or she had attended the top row, the bottom row, or "other." The participant's response was our measure of attention allocation (we tested whether the participants' responses were valid, as described in the Results section). Last, if it was a trial in which there was no target stimulus, as described next, the display read: "Press space bar to continue." Otherwise, the response display listed a column of seven sums, as shown in Figure 1. The participant's response 


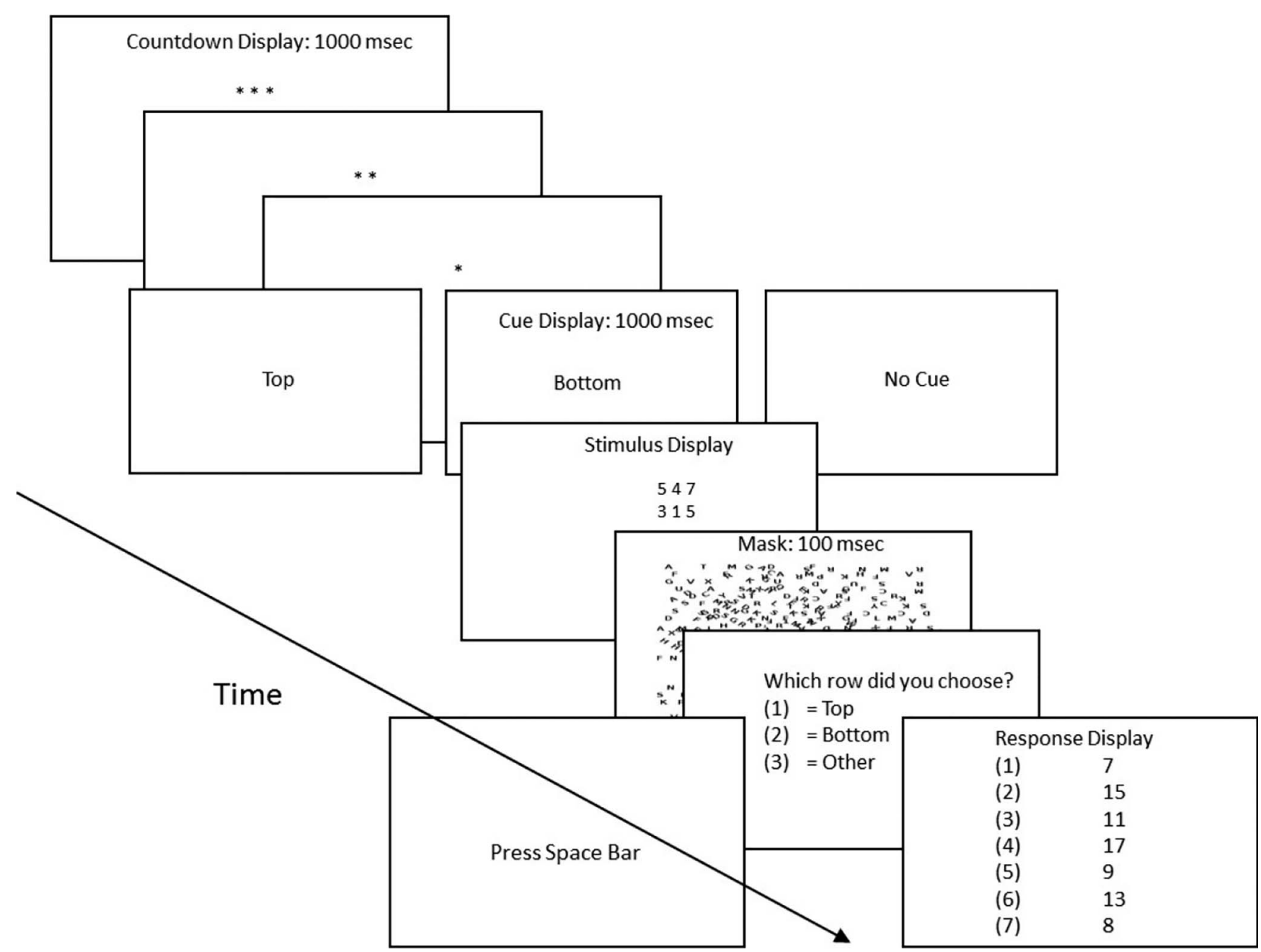

Figure 1. Schematic account of the procedure, including the durations for each step, when appropriate. Each trial began with a preparatory, countdown period, marked by three asterisks. They disappeared at a rate of $1 / \mathrm{sec}$, with a $100 \mathrm{msec}$ inter-screen interval. Following the last asterisk, a 1-sec screen displayed the words "Top" or "Bottom" when it was a cued trial or "No Cue" when it was a non-cued trial. Next was the Stimulus Display; its duration was determined by the calibration procedure-typically about $130-140 \mathrm{msec}$. This was followed by a 100 msec mask and the two response displays.

was recorded as correct or not, and it initiated the countdown period for the next trial.

Trial simulation of two independent variable timers. In the most widely used procedure for matching law choice studies, two independent timers, each stocked with a series of intervals of varying duration, determine whether a response will produce a reward. We arranged a trial version of this contingency. There were two general cases: those in which the history of the previous trial did not matter (the usual case) and those in which there was carryover from the previous trial.

Each stimulus was linked to an array of 30 numbers, spanning the interval of 1 to 30 . At the start of the trial a number was selected at random without replacement from each array. If the selected number met the criteria for setting up a correct response (described subsequently), the sum of the three digits of its linked stimulus row in the stimulus display screen appeared as one of the seven possible options in the response display screen. For the 1:1 ratio, the numbers 1 to 10 of Array 1 established that the three digits of the top row of the stimulus display was the target stimulus, and the numbers 11 to 20 of Array 2 established that the three digits of the bottom row of the stimulus display was the target stimulus. Other numbers had no effect. For the 3:1, 1:3, 9:1, and 1:9 conditions analogous strategies established whether the sum of the three digits of the top and bottom stimuli appeared in the response display, which is to say, whether the stimulus was a target. Notice that this approach yielded three possible outcomes: (1) neither stimulus had a match in the response display, (2) either the top stimulus or the bottom stimulus had a match in the response display, or (3) both the top and bottom stimulus had a match in the response display. As noted, on those trials in which the procedure did not produce a target stimulus, the response display did not 
appear, and the screen read, "Press space bar." This initiated the next trial.

The second case is when the 30-digit arrays arranged for the possibility of a correct response, but the participant failed to choose the correct sum in the response display. When this happened the target stimulus remained the target, but it displayed a new combination of digits on the next trial. In addition, it remained possible for the other stimulus to become a target (assuming it was not one already).

Although a trial procedure, the manner in which the opportunity for correct responses was arranged approximated the manner in which the opportunity for rewards are established in matching law experiments with variable interval schedules. First, the likelihood that a stimulus had become a target increased as a function of the number of trials since it last yielded a correct response. Second, given that the durations of the countdown periods and cue display were fixed, summing to $4.4 \mathrm{~s}$, the probabilities that a stimulus was a target had a temporal counterpart. For instance, in the 1:1 condition, each array arranged an overall 0.33 probability that its stimulus (row) was the target, so that the expected temporal interval from the last correct response was 13.2 s. For the 3:1 and 1:3 conditions, the expected overall average times for the corresponding cases were 8.8 and $26.4 \mathrm{~s}$ (and vice versa), and in the 9:1 and 1:9 conditions, the expected average times were 7.4 and $66.6 \mathrm{~s}$ (and vice versa). Third, once one of the two stimuli became a target, it remained so until the participant correctly identified it in the response display. Thus, in a manner analogous to how rewards set up in laboratory matching law studies, the likelihood that a stimulus was the target increased over time and once a stimulus became a target it remained so until correctly identified.

Stimulus properties and size relative to the fovea. Each digit of the stimulus display measured approximately $0.18 \mathrm{~cm} \times$ $0.32 \mathrm{~cm}$ and each row of three digits measured $0.71 \mathrm{~cm} \times 0.32 \mathrm{~cm}$, with a space of $0.24 \mathrm{~cm}$ between each row. Thus, depending on the participant's viewing distance: the entire stimulus array subtended visual angles of $0.63 \times 0.78$ to $0.95 \times 1.17$ degrees, each digit subtended visual angles of about $0.18 \times 0.28$ to $0.27 \times 0.43$ degrees, each row subtended angles of about $0.63 \times 0.28$ to $0.95 \times 0.43$ degrees, and the border between the two rows subtended visual angles of about $0.63 \times 0.21$ to $0.95 \times 0.32$ degrees. Visual angles of less than 5.0 degrees are described as "small" or "narrow" (De Cesarei and Codispoti, 2008; Goolkasian, 1991). This judgment is based on the sizes of the fovea and foveola, which, according to standard texts, are approximately 5.0 and 1.2 degrees, respectively (Wandell, 1995). Thus, the entire stimulus array (top and bottom stimulus together) were well within the view of a fixed gaze.

Session structure. Each session was 132 trials long: 22 were cued and 110 were noncued. Eleven of the cued trials occurred in the first half of the session. After Trial 66, the participants had the opportunity to take a break of up to 3 minutes. The purpose of including some cued trials in the experimental session was to check whether the calibration procedure provided the participants enough time to add correctly the three digits when they knew ahead of time which stimulus was the target-and by extension, to determine whether they had enough time to respond correctly on uncued trials when they had attended the target stimulus. In the cued trials, the top and bottom stimuli were equally likely to be the target.
Departures from standard practice. We should point out three features of the present study that are atypical. In matching law studies, researchers typically evaluate the relationship between choice proportions and the obtained reward proportions. However, choice proportions can affect the obtained reward proportions. For example, if all choices went to just one reward source then the allocation of choices would perfectly match the allocation of rewards (both equaling 100\%), but it would be unclear as to whether the independent variable was choice or the consequences of choice. Accordingly, our approach was to evaluate attention allocation as a function of the experimentally arranged number of trials that the top row stimulus was the target relative to the experimentally arranged number of trials that the bottom row stimulus was the target. This provides a less ambiguous, more informative account of the relations between attention and correct responses.

Second, the consequence of attending to the target stimulus was a correct answer (assuming that the three digits were added together correctly). The instructions described what counted as a correct answer, and participants were told to get as many correct answers as possible. However, the procedure did not provide explicit feedback as to whether a response was correct or not. Thus, the consequences for attending to a stimulus, like attention itself, were covert.

Third, in our previous attention studies, we inferred the allocation of attention and the correct guess rates by solving linear and quadratic equations that modeled performance. In this version of the procedure, we simply asked the participants which stimulus they had attended to. As shown in the Results section, the participants' responses were valid.

\section{Results}

We aggregated the results into half session units for each of the two sessions of each of the five conditions. Table 1 lists the main T1, AQ:2 findings. Here and throughout, the results are for the uncued trials, unless stated otherwise. The second column lists the average exposure times, which were determined by the calibration procedure. Averaging across the five conditions, the overall average exposure time in Session 1 was 156.7 ms (with a minimum and maximum of $70 \mathrm{~ms}$ and $370 \mathrm{~ms}$ ), and in Session 2 the average was $150.2 \mathrm{~ms}$ (with a minimum and maximum of $50 \mathrm{~ms}$ and $275 \mathrm{~ms}$ ). The third column lists the average percentage of trials that the participants correctly identified the sum of the three digits in the top row stimulus. The third column lists the average percentage of trials the participants attended the top row stimulus. And the fourth column lists the average number of times that attention switched from one stimulus to the other in each 55-trial block. In each condition, attention shifted to better approximate the percentage of trials that the top stimulus was the target. Figure 2 shows this trend $\mathbf{F} 2$ more clearly.

Figure 2 plots the results as ratios according to Equation 2 for each half session. In contrast to Table 1, which lists the obtained percentage of correct top stimulus identifications, the $x$-axis is the ratio of the number of times the top stimulus was the assigned target relative to the number of times the bottom stimulus was the assigned target. As noted in the introductory paragraphs, this seemed to us a more rigorous test of the matching law predictions for the procedure used in this study. The equations list the best 
Table 1

Summary of Key Findings on Uncued Trials

\begin{tabular}{|c|c|c|c|c|}
\hline Condition & $\begin{array}{l}\text { Stimulus exposure } \\
\text { time (ms) }\end{array}$ & $\begin{array}{l}\text { Percentage of trials correct } \\
\text { top response }\end{array}$ & $\begin{array}{l}\text { Percentage of trials participants } \\
\text { attended top stimulus }\end{array}$ & $\begin{array}{l}\text { Number of trials attention } \\
\text { switched between stimuli }\end{array}$ \\
\hline \multicolumn{5}{|l|}{ One to nine } \\
\hline Session 1,1 & $140.5(38.33)$ & $10.0(6.0)$ & $33.0(15.0)$ & $14.7(7.7)$ \\
\hline Session 1,2 & & $10.0(3.0)$ & $25.0(22.0)$ & $11.4(6.2)$ \\
\hline Session 2,1 & $154.0(54.2)$ & $9.0(5.0)$ & $25.0(21.0)$ & $8.6(7.2)$ \\
\hline Session 2, 2 & & $9.0(4.0)$ & $15.0(24.0)$ & $6.4(6.8)$ \\
\hline \multicolumn{5}{|l|}{ One to three } \\
\hline Session 1,1 & $159.2(53.9)$ & $28.0(14.0)$ & $44.0(27.0)$ & $17.0(11.8)$ \\
\hline Session 1,2 & & $27.0(14.0)$ & $40.0(27.0)$ & $20.4(15.1)$ \\
\hline Session 2,1 & $154.6(76.1)$ & $32.0(13.0)$ & $47.0(24.0)$ & $17.6(11.1)$ \\
\hline Session 2, 2 & & $29.0(15.0)$ & $38.0(25.0)$ & $17.7(10.9)$ \\
\hline \multicolumn{5}{|l|}{ One to one } \\
\hline Session 1,1 & $152.2(39.4)$ & $56.0(11.0)$ & $57.0(17.0)$ & $20.9(12.6)$ \\
\hline Session 1,2 & & $53.0(10.0)$ & $54.0(15.0)$ & $22.4(11.1)$ \\
\hline Session 2,1 & $152.2(42.0)$ & $51.0(7.0)$ & $59.0(10.0)$ & $26.7(10.5)$ \\
\hline Session 2,2 & & $54.0(11.0)$ & $55.0(14.0)$ & $27.0(12.6)$ \\
\hline \multicolumn{5}{|l|}{ Three to one } \\
\hline Session 1,1 & $113.6(39.7)$ & $74.0(15.0)$ & $67.0(19.0)$ & $14.2(6.6)$ \\
\hline Session 1,2 & & $78.0(8.0)$ & $66.0(26.0)$ & $13.7(9.1)$ \\
\hline Session 2, 1 & $124.7(34.2)$ & $79.0(11.0)$ & $74.0(25.0)$ & $12.7(10.0)$ \\
\hline Session 2,2 & & $80.0(7.0)$ & $77.0(24.0)$ & $13.5(9.5)$ \\
\hline \multicolumn{5}{|l|}{ Nine to one } \\
\hline Session 1,1 & $195.5(37.8)$ & $89.0(8.0)$ & $68.0(22.0)$ & $9.6(6.4)$ \\
\hline Session 1,2 & & $89.0(7.0)$ & $73.0(30)$. & $8.7(6.9)$ \\
\hline Session 2, 1 & $206.0(53.8)$ & $92.0(27.0)$ & $93.0(14.0)$ & $6.5(7.5)$ \\
\hline Session 2, 2 & & $92.0(29.0)$ & $91.0(28.0)$ & $5.6(5.0)$ \\
\hline
\end{tabular}

Note. Means and standard deviations (in parentheses). Session 1, 1 and 1, 2 refer to the first and second half of Session 1. Session 2, 1 and 2, 2 refer to the first and second half of Session 2.

fitting coefficients (intercepts and slopes), along with the goodness of fit proportions. The coordinates are logarithmic (in keeping with Equation 2); the curves circumscribing the linear fits are the $95 \%$ confidence intervals.

The matching law predicted the relationship between the overall programmed rates that the top and bottom stimuli were the target stimuli and the overall division of attention between the top and bottom stimuli. The slopes of the best fitting matching line systematically increased as a function of experience with the procedure, falling just short of 1.0, which is the pattern observed in behavioral studies (e.g., Todorov et al., 1983). The intercepts were positive. Since the data were plotted as ratios with the top stimulus as the numerator, this means that there was a bias for the top row stimulus. For instance, most of the participants in the $1: 1$ condition favored the top stimulus, even though the bottom stimulus was just as likely to provide the information needed for a correct response.

Figure 3 shows the trial-to-trial frequency of attentional shifts between the top and bottom stimuli as a function of the overall division of attention between the top and bottom stimuli. For purposes of context and comparison, this figure also shows the rates at which pigeons switched between two food sources in a study that was analogous to the present one (Heyman, 1979). In both experiments, the ratios of arranged reinforcers (correct answers and grain) varied from 1:9 to 9:1, and in both the arranged absolute reinforcement rates, summing across the two options, was constant across conditions. The data are plotted as percentages of the transition rate in the $1: 1$ condition.

The analogous conditions produced analogous results. In the present study, attention shifted between the two stimuli most frequently in the $1: 1$ condition, least frequently in the $1: 9$ and $9: 1$ conditions, and at intermediate frequencies in the $3: 1$ and $1: 3$ conditions. Analogously, the pigeons switched between the two food sources most frequently in the 1:1 food condition, least frequently in the 1:9 and 9:1 food condition, and at intermediate frequencies in the $3: 1$ and $1: 3$ conditions.

Figure 4 shows the percentage of correct responses under four $\mathbf{F 4}$ different conditions. The first two bars are the percentage of correct responses on trials in which the participant did not attend the target stimulus. The expected value, assuming no usable knowledge of the target stimulus, is $14.3 \%$. The average observed value was $15.0 \%$. The third bar is the percentage of correct responses on the cued trials in the experimental sessions, and the fourth bar is the percentage of correct responses on the uncued trials in which the particpants attended the target stimulus. On cued trials, participants identified the target sum correctly $89 \%$ of the time. On uncued trials in which the participants attended the target stimulus, they identified the target sum correctly $95 \%$ of the time. In other words, on uncued trials the participants reliably reported which stimulus they had attended to and made few errors adding up the three digits.

\section{Discussion}

\section{The Overall Allocation of Attention and Trial-to-Trial Shifts in Attention}

Figure 2 summarizes the molar results. It shows that the generalized matching law equation described the allocation of attention 


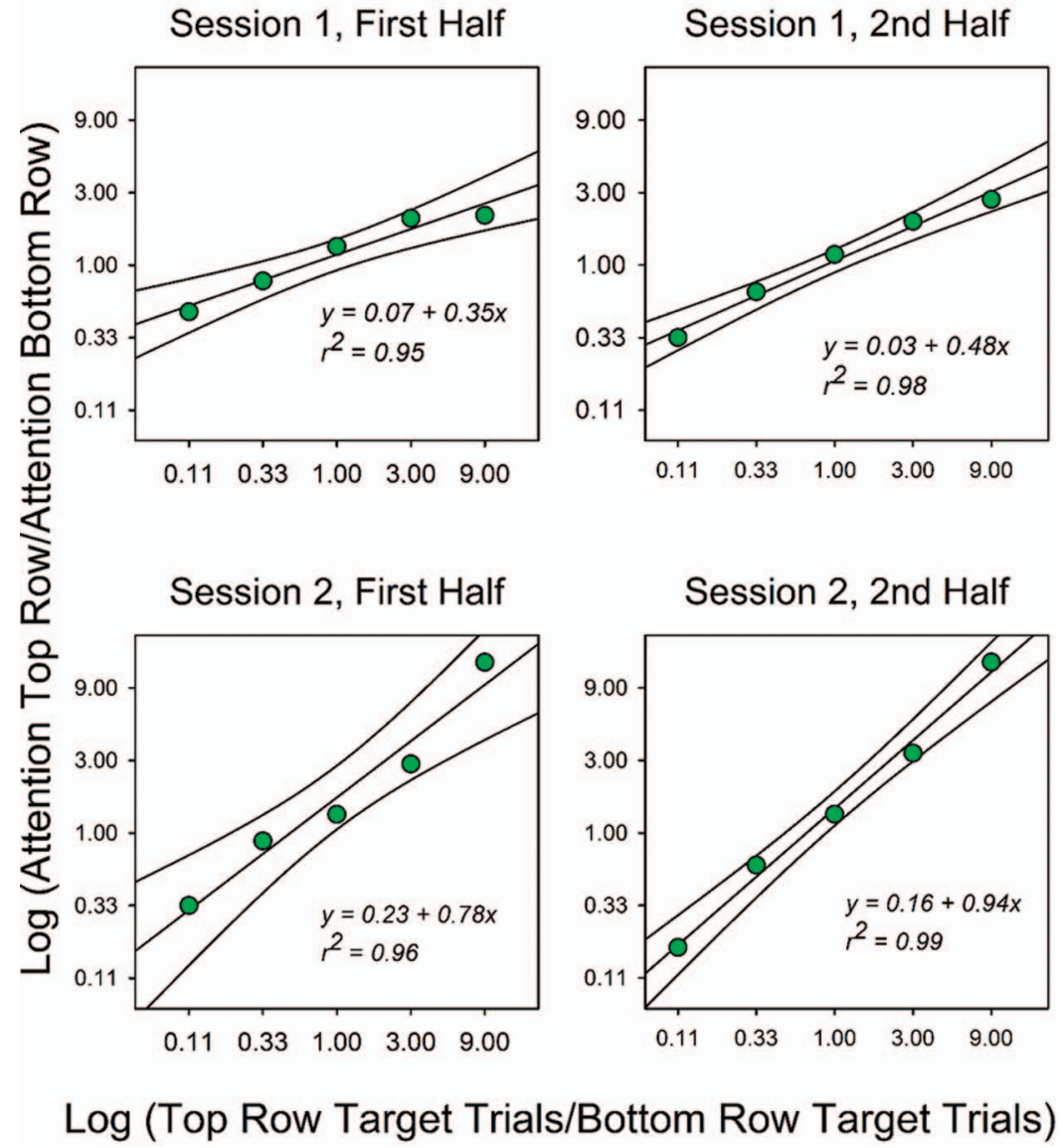

Figure 2. Attention allocation ratios as a function of the number of trials the top stimulus was the target relative to the number of trials the bottom stimulus was the target (in logarithmic coordinates). The data points are group averages, and the bowed lines trace out the $95 \%$ confidence intervals. The intercepts and slopes in the equations are the best fitting parameters for the generalized matching law. See the online article for the color version of this figure.

at least as well as it describes behavior in choice experiments. It accounted for $95 \%$ to $99 \%$ of the variance in attention allocation ratios, and, as observed in behavioral studies (e.g., Todorov et al., 1983), the slopes of the fitted functions - which reflect the influence of variation in reward ratios-increased as a function of exposure to the procedure, approaching a value of 1.0. The intercepts were consistently greater than 0.0 , implying a bias in favor of the top row stimulus.

Figure 3 summarizes the trial-to-trial shifts in attention along with the changeover rates from a pigeon study. As described in the preceding text, the procedure in the pigeon experiment was analogous to the one used in the present study: the overall programmed rates of reinforcement were constant (counting both variable interval schedules), whereas the relative rates varied over the range of $1: 9$ to $9: 1$, just as in the present study. In both experiments, transition rates were greatest at about a 50:50 allocation of the activity in question and then trailed off symmetrically as choice proportions became more extreme. As explained elsewhere, this is precisely the expected (and observed) pattern if the moment-tomoment probabilities of a transition are stationary, as in a simple, two-state Markov processes (Heyman, 1979, 1982). Thus, the relationship between moment-to-moment shifts in attention and the overall division of attention followed the quantitative pattern observed in behavioral studies in which the probability of switching from one food source to the other was relatively constant over time.

\section{Tests of Our Key Assumptions}

We have assumed that in each trial, (1) the participants' selfreports faithfully reflected which stimulus they attended to, (2) that if the participant attended to a stimulus, he or she correctly added up the digits, and (3) that participants had no usable knowledge of the stimulus that they did not attend to. The findings supported these assumptions. On uncued trials, accuracy rate was $95 \%$ for the stimulus that the participants said they had attended to. In contrast, accuracy rate was nearly chance ( 15.0 vs. $14.3 \%$ ) on trials in which attention was allocated to the stimulus that did not provide the correct information. These numbers closely approximate the performance of an ideal participant who on any given trial attended to one stimulus, added the three digits correctly, but did not attend to both stimuli. 


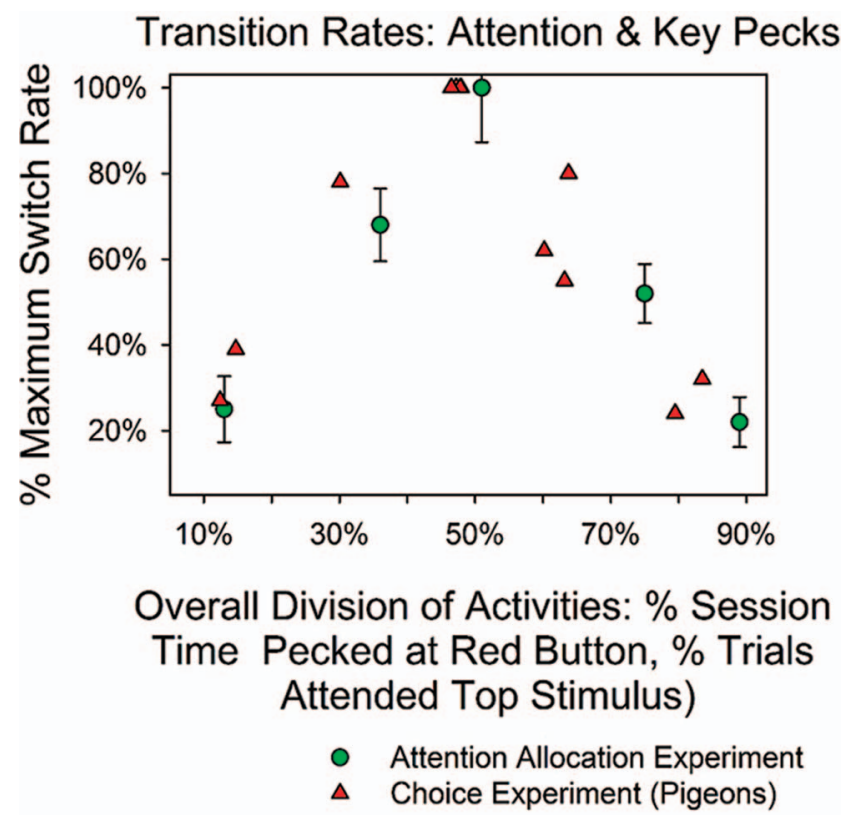

Figure 3. The filled circles show shifts in attention from the last half of the second session. The filled triangles show changeover rate for four pigeons, averaged across the last five sessions of each condition. The results from the present experiment are group averages, and the error bars indicate the standard errors. For the data from the present study, the $x$-axis lists the overall division of attention between the two stimuli (as percentages). For the pigeon experiment, the $x$-axis lists the overall division of time spent at each food source. See the online article for the color version of this figure.

The results also replicate our earlier results (Heyman et al., 2016). In the initial study, the correct guess rate was $16.2 \%$ and the percentage of trials in which the participants correctly identified the three-digit sum on cued trials was $90 \%$. However, in contrast to the present experiment, the correct guess rate was one of the solutions to the mathematical model that calculated how attention was allocated. That is, correct guess rates determined by selfreport and correct guess rates determined by mathematical inference differed by no more than an average of $1.2 \%$.

\section{Relations With Previous Research}

The overall attention allocation measures (see Figure 2) and the rate at which attention shifted between the two stimuli on a trial-to-trial basis closely replicate the results from typical laboratory matching law studies, differences in species and type of reward notwithstanding. However, it is also the case that the results in this study differed from those reported in the behavioral literature to some degree.

First, in each of the four conditions summarized in Figure 2, the intercept for the best fitting matching law equation was positive. This means that there was a bias for the top stimulus. For example, in the $1: 1$ condition, the average allocation ratio was $1.28 / 1.00$, even though the programmed likelihood that the top stimulus was the target was precisely $1: 1$. In behavioral studies, nonzero intercepts reflect a side bias or a qualitative difference in the competing rewards. In this experiment, there were no "sides" or explicit rewards that could differ. However, in studies of cognition that also arranged brief exposures to $2 \times 4$ stimulus matrices, participants were more likely to report elements from the first row (e.g., Sperling, 1960; Lass, Yan, Chen, Becker, \& Luer, 2008), an effect that Lass et al. attribute to reading habits. This interpretation is consistent with recent research programs that emphasize that in laboratory studies, as in everyday life, attention is guided by a host of factors, including implicit, long-term cognitive patterns, such as reading text from top to bottom (see Chelazzi et al., 2018; Todd \& Manaligod, 2018)

Second, in trials in which participants reported attending the stimulus that was not the target, they were correct on $15 \%$ of the trials. This result closely approximates the expected guess rate (1/7), which implies that that the participants had virtually no usable knowledge of the unattended stimulus. This is consistent with "winner take all" attention models (see, e.g., Zénon, Ben Hamed, Duhamel, \& Olivier, 2009). However, as noted in the Limitations section, there are good reasons to believe that the winner take all aspect of the present results emerged either as a function of repeated exposure to the procedure and/or late in the perceptual/cognitive processes that mediated the participants' choices in the response display stage of the experiment.

\section{Limitations}

In the introductory paragraphs, we pointed out that our procedure and measures do not locate the constraints on cognition. Viewed in isolation, the results are equally compatible with attention models that emphasize early selection processes (e.g., Treisman, 1969) and those that emphasize late selection processes (e.g., Duncan, 1980; Lavie, Hirst, de Fockert, \& Viding, 2004). However, the research literature suggests that it is more likely that late selection processes played a more important role First, in a much referenced study that used stimuli that were roughly the same size as those in the present experiment, Eriksen and Hoffman (1973) 

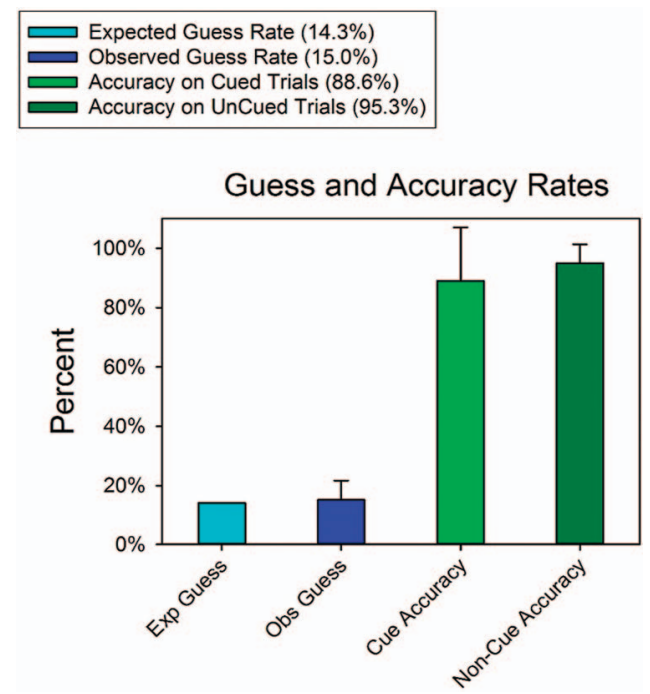

Figure 4. The probabilities of a correct response on cued and uncued trials. The first bar is the expected percentage of correct responses for the case that the participant attended the incorrect stimulus and had no usable knowledge of the contents of the correct stimulus (i.e., the expected correct guess rate). The second bar is the observed correct guess rate. The third bar is the percentage of correct responses in cued trials. The fourth bar is the percentage of correct responses on uncued trials in which the participants reported attending the designated target (correct) stimulus. See the online article for the color version of this figure.

found evidence that there is an irreducible limit on the "size" of attentional focus that corresponds to 1.0 degree of visual angle. If this is correct, not only did a gaze encompass both the top and bottom stimuli but so did visual-spatial attention in the initial stimulus display. Second, in support of this suggestion, numerous studies show that in simple attention tasks (such as identifying a particular letter or slanted line), participants are capable of attending two spatial locations that are further apart than the top and bottom stimuli in the present study (e.g., Jonides, 1983; Bay \& Wyble, 2014; Wolfe, 1992). Thus, it is reasonable to suppose that in this study participants were capable of attending to both the top and bottom stimuli in the stimulus display stage of each trial. Why then, did the participants have no usable knowledge of the unattended to stimulus when selecting a response in the final, response display stage of each trial? The simplest account is that the unattended stimulus was not as fully encoded or disappeared from working memory in the process of adding together the three digits of the attended row. In addition, it is possible that as the participants learned which stimulus was more likely to be the target, suppressive or inhibitory processes were triggered that limited early processing of the unattended stimulus (e.g., Bridwell \& Srinivasan, 2012; Wuhr \& Frings, 2008). For instance, a priming procedure might reveal some knowledge of the unattended stimulus and would perhaps be more likely to do so in the early trials of the initial session. Alternatively, it would be of interest to test whether rapid serial visual presentation methods in which the statistical properties of the stimuli, rather than a visual cue, signaled the target yielded results similar to those presented here or those demonstrating parallel processing of more than one target (e.g., Bay \& Wyble, 2014), and whether differences, should they exist, emerged as a function of practice.

A more general limitation is one that attends virtually all research on the matching law relations. In the present procedure, and in the more typical procedures in which the possibility of reinforcement is continuous and not trial-by-trial, the moment to moment probabilities of reward change dynamically as a function of moment-to-moment choices. These dynamics are ignored by Equations 1 and 2 and are usually ignored in research reports. In support of this approach are studies that explicitly tracked the moment-to-moment response patterns (e.g., Heyman, 1979; Nevin, 1969). There was little or no evidence of moment-to-moment reward probability tracking. Indeed, in one case a simple two-state Markov model fit the results (Heyman, 1979). Thus, it is not obvious that more molecular analyses would prove fruitful.

\section{Concluding Remarks}

The matching law was originally formulated as a quantitative summary of the relations between voluntary behaviors and their consequences. In the original studies, the consequences were events that were established reinforcers, such as grain for hungry pigeons and chow for hungry rats. In this experiment, the consequences were the participants' impressions that they had made a correct response-there was no feedback. By the criteria used in behavioral studies, such impressions functioned as reinforcers. This suggests that self-generated self-appraisal ("I found the sum of the three digits") was functionally similar to environmental generated, tangible rewards. In turn, this raises the possibility that covert positive self-regard and positive consummatory events share some common, underlying factors.

The allocation of cognitive resources and the allocation of overt behavior are similar in that both involve the problem of how to manage limited resources. This point suggests that if the matching law describes the allocation of choices, it should also describe how individuals divide their attention between competing stimuli. The results summarized by Figures 2 and 3 support this line of reasoning. The matching law described the allocation of covert cognitive processes as well as it describes the allocation of key pecks, lever presses, and the other behavioral indices experimenters have used to investigate choice. What remains to be established is the nature of the cognitive constraints that limited correct responses to just one of the two stimuli on each trial. Did they take place in the initial response to the stimulus display, did they take place later in the course of adding together the three digits, or both? Similarly, at what stage or stages did the constraints operate as predicted by the matching law? Answering these questions will likely prove no small task; however, prior to conducting our experiment, we did not know that these questions needed to be asked.

\section{Context}

Heyman's introductory psychology course motivated our experiment. The readings suggested parallels between attention allocation and choice (e.g., Corbetta, Miezin, Dobmeyer, Shulman, \& Petersen, 1990; Sperling, 1960). To test this idea, we developed a mathematical model and experimental procedure for calculating the allocation of covert visual attention; eye movements played no role (Heyman et al., 2016, 2017). The results were systematic, but 
the procedure was limited in that the results that-were compatible with any one of several quantitative choice theories. These ambiguities motivated the present study. We modified the procedure so that it would unambiguously test whether the matching law applied. Although shifts in attention were covert, and the procedure did not provide explicit reinforcers, the matching law equation described the division of attention between two small, briefly presented stimuli as accurately as it describes the allocation of key pecking between two illuminated disks in hungry pigeons.

\section{References}

Anderson, B. A., \& Yantis, S. (2013). Persistence of value-driven attentional capture. Journal of Experimental Psychology: Human Perception and Performance, 39, 6-9. http://dx.doi.org/10.1037/a0030860

Arvanitogiannis, A., \& Shizgal, P. (2008). The reinforcement mountain: Allocation of behavior as a function of the rate and intensity of rewarding brain stimulation. Behavioral Neuroscience, 122, 1126-1138. http:// dx.doi.org/10.1037/a0012679

Baum, W. M. (1974). On two types of deviation from the matching law: Bias and undermatching. Journal of the Experimental Analysis of Behavior, 22, 231-242. http://dx.doi.org/10.1901/jeab.1974.22-231

Baum, W. M. (1979). Matching, undermatching, and overmatching in studies of choice. Journal of the Experimental Analysis of Behavior, 32, 269-281. http://dx.doi.org/10.1901/jeab.1979.32-269

Bay, M., \& Wyble, B. (2014). The benefit of attention is not diminished when distributed over two simultaneous cues. Attention, Perception \& Psychophysics, 76, 1287-1297. http://dx.doi.org/10.3758/s13414-0140645-z

Borrero, J. C., \& Vollmer, T. R. (2002). An application of the matching law to severe problem behavior. Journal of Applied Behavior Analysis, 35, 13-27. http://dx.doi.org/10.1901/jaba.2002.35-13

Bridwell, D. A., \& Srinivasan, R. (2012). Distinct attention networks for feature enhancement and suppression in vision. Psychological Science, 23, 1151-1158. http://dx.doi.org/10.1177/0956797612440099

Cero, I., \& Falligant, J. M. (2020). Application of the generalized matching law to chess openings: A gambit analysis. Journal of Applied Behavior Analysis, 53, 835-845. http://dx.doi.org/10.1002/jaba.612

Chelazzi, L., Bisley, J. W., \& Bartolomeo, P. (2018). The unconscious guidance of attention. Cortex, 102, 1-5. http://dx.doi.org/10.1016/j .cortex.2018.02.002

Chelazzi, L., Perlato, A., Santandrea, E., \& Della Libera, C. (2013). Rewards teach visual selective attention. Vision Research, 85, 58-72. http://dx.doi.org/10.1016/j.visres.2012.12.005

Conger, R., \& Killeen, P. (1974). Use of concurrent operants in small group research: A demonstration. Pacific Sociological Review, 17, $399-$ 416. http://dx.doi.org/10.2307/1388548

Corbetta, M., Miezin, F. M., Dobmeyer, S., Shulman, G. L., \& Petersen, S. E. (1990). Attentional modulation of neural processing of shape, color, and velocity in humans. Science, 248, 1556-1559. http://dx.doi .org/10.1126/science. 2360050

Davison, M., \& McCarthy, D. (1988). The matching law: A research review. Hillsdale, NJ: Lawrence Erlbaum.

De Carlo, L. T., \& Abramson, C. I. (2012). "Time allocation in carpenter ants (Camponotus herculeanus)": Correction to De Carlo \& Abramson (1989). Journal of Comparative Psychology, 126, 346. http://dx.doi.org/ 10.1037/a0030304

De Cesarei, A., \& Codispoti, M. (2008). Fuzzy picture processing: Effects of size reduction and blurring on emotional processing. Emotion, 8, 352-363. http://dx.doi.org/10.1037/1528-3542.8.3.352

Duncan, J. (1980). The locus of interference in the perception of simultaneous stimuli. Psychological Review, 87, 272-300. http://dx.doi.org/10 $.1037 / 0033-295 X .87 .3 .272$
Eriksen, C. W., \& Hoffman, J. E. (1973). The extent of processing of noise elements during selective encoding from visual displays. Perception \& Psychophysics, 14, 155-160. http://dx.doi.org/10.3758/BF03198630

Falligant, J. M., Boomhower, S. R., \& Pence, S. T. (2016). Application of the generalized matching law to point-after-touchdown conversions and kicker selection in college football. Psychology of Sport and Exercise, 26, 149-153. http://dx.doi.org/10.1016/j.psychsport.2016.07.006

Foxall, G. R., James, V. K., Oliveira-Castro, J. M., \& Ribier, S. (2010). Product substitutability and the matching law. The Psychological Record, 60, 185-216. http://dx.doi.org/10.1007/BF03395703

Goolkasian, P. (1991). The effect of size on the perception of ambiguous figures. Bulletin of the Psychonomic Society, 29, 161-164. http://dx.doi .org/10.3758/BF03335224

Grace, R. C., \& Hucks, A. D. (2013). The allocation of operant behavior. In G. J. Madden, W. V. Dube, T. D. Hackenberg, G. P. Hanley, \& K. A. Lattal (Eds.), APA handbook of behavior analysis, Vol. 1: Methods and principles (pp. 307-337). Washington, DC: American Psychological Association. http://dx.doi.org/10.1037/13937-014

Gray, L. N., Stafford, M. C., \& Tallman, I. (1991). Rewards and punishments in complex human choices. Social Psychology Quarterly, 54, 318-329. http://dx.doi.org/10.2307/2786844

Herrnstein, R. J. (1970). On the law of effect. Journal of the Experimental Analysis of Behavior, 13, 243-266. http://dx.doi.org/10.1901/jeab.1970 .13-243

Herrnstein, R. J., Rachlin, H., \& Laibson, D. I. (Eds.). (1997). The matching law: Papers in psychology and economics. Cambridge, MA: Harvard AQ: 7 University Press.

Heyman, G. M. (1979). A Markov model description of changeover probabilities on concurrent schedules. Journal of the Experimental Analysis of Behavior, 31, 41-51. http://dx.doi.org/10.1901/jeab.1979.31-41

Heyman, G. M. (1982). Is time allocation elicited behavior? In M. Commons, R. Herrnstein, \& H. Rachlin (Eds.), Quantitative analyses of behavior: Vol. 2. Matching and maximizing accounts (pp. 459-490). Cambridge, MA: Ballinger Press.

Heyman, G. M., Grisanzio, K. A., \& Liang, V. (2016). Introducing a method for calculating the allocation of attention in a cognitive "twoarmed bandit" procedure: Probability matching gives way to maximizing. Frontiers in Psychology, 7, 223. http://dx.doi.org/10.3389/fpsyg .2016 .00223

Heyman, G. M., \& Luce, R. D. (1979). Operant matching is not a logical consequence of reinforcement rate maximization. Animal Learning \& Behavior, 7, 133-140. http://dx.doi.org/10.3758/BF03209261

Heyman, G. M., Montemayor, J., \& Grisanzio, K. A. (2017). Dissociating attention and eye movements in a quantitative analysis of attention allocation. Frontiers in Psychology, 8, 715. http://dx.doi.org/10.3389/ fpsyg.2017.00715

Houston, A. (1986). The matching law applies to wagtails' foraging in the wild. Journal of the Experimental Analysis of Behavior, 45, 15-18. http://dx.doi.org/10.1901/jeab.1986.45-15

Houston, A. I., McNamara, J. M., \& Steer, M. D. (2012). Do we expect natural selection to produce rational behaviour? In A. K. Seth, T. J. Prescott, \& J. J. Bryson (Eds.), Modelling natural action selection (pp. 12-36). New York, NY: Cambridge University Press.

Jiang, Y. V. (2018). Habitual versus goal-driven attention. Cortex, 102, 107-120. http://dx.doi.org/10.1016/j.cortex.2017.06.018

Jonides, J. (1983). Further toward a model of the mind's eye's movement. Bulletin of the Psychonomic Society, 21, 247-250. http://dx.doi.org/10 .3758/BF03334699

Kubanek, J. (2017). Optimal decision making and matching are tied through diminishing returns. Proceedings of the National Academy of Sciences of the United States of America, 114, 8499-8504. http://dx.doi .org/10.1073/pnas.1703440114

Lass, U., Yan, S., Chen, G., Becker, D., \& Lüer, G. (2008). Position effects in encoding briefly exposed item matrices: Evidence for a reading bias 
or merely a matter of the selection criterion? Psychological Research, 72, 641-647. http://dx.doi.org/10.1007/s00426-008-0169-z

Lavie, N., Hirst, A., de Fockert, J. W., \& Viding, E. (2004). Load theory of selective attention and cognitive control. Journal of Experimental Psychology: General, 133, 339-354. http://dx.doi.org/10.1037/00963445.133.3.339

Lee, J., \& Shomstein, S. (2014). Reward-based transfer from bottom-up to top-down search tasks. Psychological Science, 25, 466-475. http://dx .doi.org/10.1177/0956797613509284

Le Pelley, M. E., Vadillo, M., \& Luque, D. (2013). Learned predictiveness influences rapid attentional capture: Evidence from the dot probe task. Journal of Experimental Psychology: Learning, Memory, and Cognition, 39, 1888-1900. http://dx.doi.org/10.1037/a0033700

Loewenstein, Y., Seung, H. S., \& Newsome, W. T. (2006). Operant matching is a generic outcome of synaptic plasticity based on the covariance between reward and neural activity. Proceedings of the National Academy of Sciences of the United States of America, 103, 15224-15229. http://dx.doi.org/10.1073/pnas.0505220103

Nevin, J. A. (1969). Interval reinforcement of choice behavior in discrete trials. Journal of the Experimental Analysis of Behavior, 12, 875-885. http://dx.doi.org/10.1901/jeab.1969.12-875

Raymond, J. E., \& O'Brien, J. L. (2009). Selective visual attention and motivation: The consequences of value learning in an attentional blink task. Psychological Science, 20, 981-988. http://dx.doi.org/10.1111/j .1467-9280.2009.02391.x

Rees, C., \& Winfree, L. T., Jr. (2017). Social learner decision making: Matching theory as a unifying framework for recasting a general theory. In W. Bernasco, J.-L. Van Gelder, \& H. Elffers (Eds.), The Oxford handbook of offender decision making (pp. 268-300). New York, NY: Oxford University Press.

Shahan, T. A., \& Podlesnik, C. A. (2006). Divided attention performance and the matching law. Learning \& Behavior, 34, 255-261. http://dx.doi .org/10.3758/BF03192881

Shanks, D. R., Tunney, R. J., \& McCarthy, J. D. (2002). A re-examination of probability matching and rational choice. Journal of Behavioral Decision Making, 15, 233-250. http://dx.doi.org/10.1002/bdm.413

Shaw, M. L., \& Shaw, P. (1977). Optimal allocation of cognitive resources to spatial locations. Journal of Experimental Psychology: Human Perception and Performance, 3, 201-211. https://doi-org.ezp-prod1.hul. harvard.edu/10.1037/0096-1523.3.2.201
Small, D. M., Gitelman, D., Simmons, K., Bloise, S. M., Parrish, T., \& Mesulam, M. M. (2005). Monetary incentives enhance processing in brain regions mediating top-down control of attention. Cerebral Cortex, 15, 1855-1865. http://dx.doi.org/10.1093/cercor/bhi063

Soltani, A., \& Wang, X. J. (2006). A biophysically based neural model of matching law behavior: Melioration by stochastic synapses. The Journal of Neuroscience: The Official Journal of the Society for Neuroscience, 26, 3731-3744. http://dx.doi.org/10.1523/JNEUROSCI.5159-05.2006

Sperling, G. (1960). The information available in brief visual presentations. Psychological Monographs, 74, 1-29. http://dx.doi.org/10.1037/ h0093759

Todd, R. M., \& Manaligod, M. G. M. (2018). Implicit guidance of attention: The priority state space framework. Cortex, 102, 121-138. http://dx.doi.org/10.1016/j.cortex.2017.08.001

Todorov, J. C., de Oliveira Castro, J. M., Hanna, E. S., Bittencourt de Sa, M. C., \& Barreto, M. Q. (1983). Choice, experience, and the generalized matching law. Journal of the Experimental Analysis of Behavior, 40, 99-111. http://dx.doi.org/10.1901/jeab.1983.40-99

Treisman, A. M. (1969). Strategies and models of selective attention Psychological Review, 76, 282-299. http://dx.doi.org/10.1037/h0027242

Vollmer, T. R., \& Bourret, J. (2000). An application of the matching law to evaluate the allocation of two- and three-point shots by college basketball players. Journal of Applied Behavior Analysis, 33, 137-150. http://dx.doi.org/10.1901/jaba.2000.33-137

Wandell, B. A. (1995). Foundations of vision. Sunderland, MA: Sinauer Associates.

Wolfe, J. M. (1992). The parallel guidance of visual attention. Current Directions in Psychological Science, 1, 124-128. http://dx.doi.org/10 .1111/1467-8721.ep10769733

Wühr, P., \& Frings, C. (2008). A case for inhibition: Visual attention suppresses the processing of irrelevant objects. Journal of Experimental Psychology: General, 137, 116-130. http://dx.doi.org/10.1037/00963445.137.1.116

Zénon, A., Ben Hamed, S., Duhamel, J.-R., \& Olivier, E. (2009). Attentional guidance relies on a winner-take-all mechanism. Vision Research, 49, 1522-1531. http://dx.doi.org/10.1016/j.visres.2009.03.010

Received November 4, 2019

Revision received February 24, 2020

Accepted May 18, 2020 